\title{
Colonic Ganglioneuroma: A Rare Lesion With Extremely Different Presentations and Outcomes in Two Patients
}

\author{
Ahmed Baiomi ${ }^{a}$, b, e, Hafsa Abbas ${ }^{a, b}$, Masooma Niazia, c, Helen Remotti ${ }^{\text {, }}$, \\ Myrta Daniel ${ }^{\mathrm{a}, \mathrm{b}}$, Bhavna Balar ${ }^{\mathrm{a}, \mathrm{b}}$
}

\begin{abstract}
Ganglioneuroma $(\mathrm{GN})$ of the gastrointestinal tract is an extremely rare neuroectodermal tumor. Patients with ganglioneuromas have different presentations depending on the location, extent and size of the lesion. We present two cases of ganglioneuromas that had different clinical presentations and outcomes.
\end{abstract}

Keywords: Neuroganglioma; Colon cancer; Abdominal pain; Abdominal mass

\section{Introduction}

Colonic ganglioneuromas are neuroectodermal polyps of the gastrointestinal (GI) tract [1]. Ganglioneuroma is composed of ganglion cells, nerve fibers and Schwann sheath elements [2]. Histologically three ganglioneuromatous proliferations have been described: polypoid ganglioneuroma, ganglioneuromatous polyposis (GP) and diffuse ganglioneuromatosis (DG). Due to the rarity of this lesion, incidence of colonic ganglioneuroma is not known. A few cases have been reported in medical literature and most of them report a benign nature of the ganglioneuroma; however a few report ganglioneuroma coexisting with cancer of the colon [3-5]. However no relationship has been established between colon cancer and ganglioneuroma, and there are no guidelines available for surveillance when these two conditions coexist. Here we present two

Manuscript submitted April 2, 2021, accepted April 16, 2021

Published online June 19, 2021

aDepartment of Medicine, BronxCare Health System, 1650 Selwyn Ave, Bronx, NY 10457, USA

bDivision of Gastroenterology, BronxCare Health System, 1650 Grand Concourse, Bronx, NY 10457, USA

${ }^{\mathrm{c}}$ Division of Pathology, BronxCare Health System, 1650 Grand Concourse, Bronx, NY 10457, USA

${ }^{\mathrm{d}}$ Department of Pathology and Cell Biology, Columbia University Irving Medical Center, 630 W 168th Street, New York, NY 10032, USA

${ }^{\mathrm{e}}$ Corresponding Author: Ahmed Baiomi, Division of Gastroenterology, Department of Medicine, BronxCare Health system, Bronx, NY 10457, USA. Email: abaiomi@bronxcare.org

doi: https://doi.org/10.14740/gr1379 cases; one with GP coexisting with malignant mixed adenocarcinoma and neuroendocrine carcinoma of the colon, and another case of isolated polypoid ganglioneuroma in a patient presenting with abdominal pain.

\section{Case Reports}

\section{Case 1}

A 44-year-old male presented to the emergency department with lower abdominal pain for the past 1 month. He described the pain to be located in the right lower quadrant, aching in nature, moderate in severity, non-radiating and associated with subjective fever. He denied any other associated symptoms. He did not report change in his bowel habits. He had a past medical history of congenital cataract. He denied smoking, alcohol or drug use. On initial examination in the emergency department, the patient was afebrile, in mild distress due to pain and was hemodynamically stable. His abdominal examination revealed mild tenderness in the right lower quadrant without any peritoneal signs. The rest of the physical examination and review of systems were unremarkable. Laboratory tests are discussed in Table 1.

Initial impression was acute appendicitis. Computed tomography $(\mathrm{CT})$ scan of abdomen with intravenous contrast revealed marked thickening and fat stranding along the appendix, cecum, and ascending colon suspicious for neoplasm. Additionally, well-defined heterogeneous, hypodense parenchymal lesions were seen, measuring $14.9 \times 10.3 \times 14.3 \mathrm{~cm}$ within the right hepatic lobe and $6.8 \times 10.8 \times 10.9 \mathrm{~cm}$ within hepatic segments 2 and 3 . At this point, the possible diagnosis of colon cancer with liver metastasis was entertained. The patient underwent colonoscopy that revealed a malignant appearing, polypoid, ulcerated, large obstructing mass at the ascending colon (Fig. 1), in addition to a submucosal non-obstructing medium-sized mass with central umbilication (donut-shaped) at the hepatic flexure. He also was noted to have multiple small polyps (Fig. 2) with normal appearing mucosa throughout the colon. Histopathology of the biopsy of the mass at the ascending colon showed invasive adenocarcinoma with signet ring cell features (Fig. 3) and poorly differentiated neuroendocrine carcinoma (high-grade) (Fig. 4) at the hepatic flexure respectively. The Ki67 proliferative index was $40 \%$ (Fig. 5). Additionally, multiple pedunculated and sessile polyps histologically were ganglioneuromas (Fig. 6). Immunohistochemical 
Table 1. Initial relevant Laboratory Values on Presentation

\begin{tabular}{lll}
\hline Laboratory test & Result (normal range), day $\mathbf{1}$ & Result (normal range), day 2 \\
\hline Hemoglobin & $12.3 \mathrm{~g} / \mathrm{dL}(12-16 \mathrm{~g} / \mathrm{dL})$ & $11 \mathrm{~g} / \mathrm{dL}(12-16 \mathrm{~g} / \mathrm{dL})$ \\
Hematocrit & $36.6 \%(42-51 \%)$ & $33 \%(42-51 \%)$ \\
Leukocyte count & $13,300 / \mu \mathrm{L}(4,800-10,800 / \mu \mathrm{L})$ & $12,800 / \mu \mathrm{L}(4,800-10,800 / \mu \mathrm{L})$ \\
Platelet count & $239,000 / \mu \mathrm{L}(150,000-400,000 / \mu \mathrm{L})$ & $209,000 / \mu \mathrm{L}(150,000-400,000 / \mu \mathrm{L})$ \\
Blood urea nitrogen & $9 \mathrm{mg} / \mathrm{dL}(8-26 \mathrm{mg} / \mathrm{dL})$ & $9 \mathrm{mg} / \mathrm{dL}(8-26 \mathrm{mg} / \mathrm{dL})$ \\
Serum creatinine & $0.8 \mathrm{mg} / \mathrm{dL}(0.5-1.5 \mathrm{mg} / \mathrm{dl})$ & $0.8 \mathrm{mg} / \mathrm{dL}(0.5-1.5 \mathrm{mg} / \mathrm{dL})$ \\
Serum albumin & $3.9 \mathrm{~g} / \mathrm{dL}(3.2-4.6 \mathrm{~g} / \mathrm{dL})$ & $3.4 \mathrm{~g} / \mathrm{dL}(3.2-4.6 \mathrm{~g} / \mathrm{dL})$ \\
Serum total bilirubin & $2.1 \mathrm{mg} / \mathrm{dL}(0.2-1.1 \mathrm{mg} / \mathrm{dL})$ & $1.6 \mathrm{mg} / \mathrm{dL}(0.2-1.1 \mathrm{mg} / \mathrm{dL})$ \\
Alkaline phosphatase & $45 \mathrm{U} / \mathrm{L}(43-160 \mathrm{U} / \mathrm{L})$ & $36 \mathrm{U} / \mathrm{L}(43-160 \mathrm{U} / \mathrm{L})$ \\
Serum alanine aminotransferase & $14 \mathrm{U} / \mathrm{L}(5-40 \mathrm{U} / \mathrm{L})$ & $12 \mathrm{U} / \mathrm{L}(5-40 \mathrm{U} / \mathrm{L})$ \\
Serum aspartate transaminase & $18 \mathrm{U} / \mathrm{L}(9-36 \mathrm{U} / \mathrm{L})$ & $14 \mathrm{U} / \mathrm{L}(9-36 \mathrm{U} / \mathrm{L})$ \\
Lactic acid level & $2.0 \mathrm{mmol} / \mathrm{L}(0.5-1.6 \mathrm{mmol} / \mathrm{L})$ & $1.3 \mathrm{mmol} / \mathrm{L}(0.5-1.6 \mathrm{mmol} / \mathrm{L})$ \\
\hline
\end{tabular}

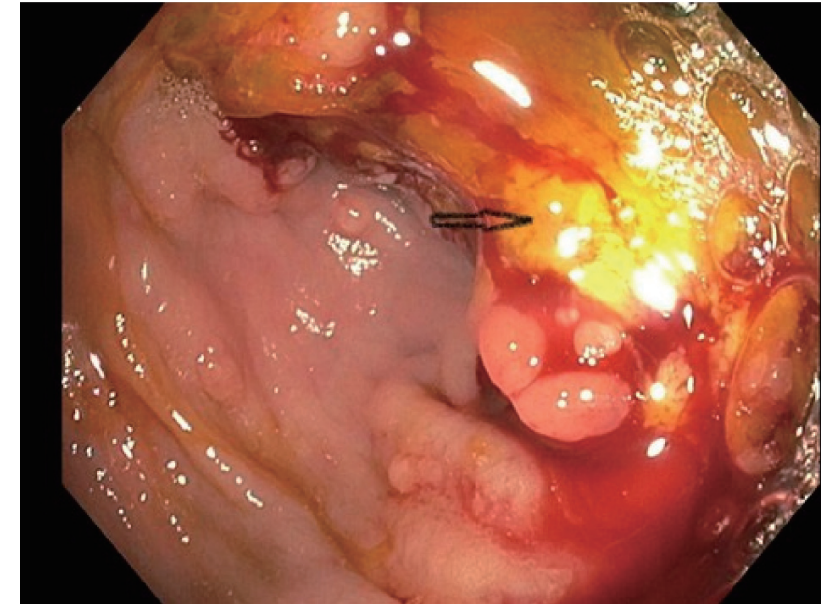

Figure 1. Colonoscopy image in the ascending colon with arrow pointing at obstructing mass.

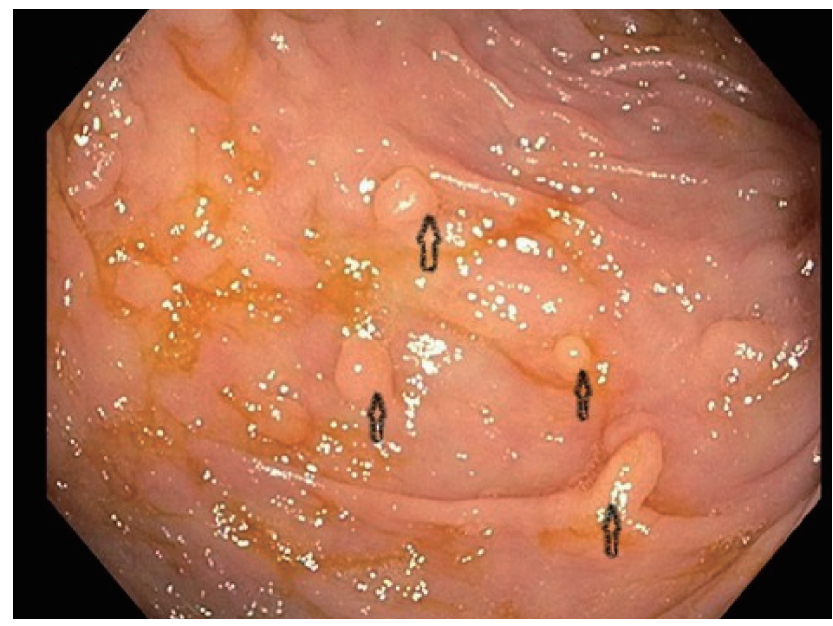

Figure 2. Colonoscopy image in the descending colon with multiple arrows pointing at multiple polyps.

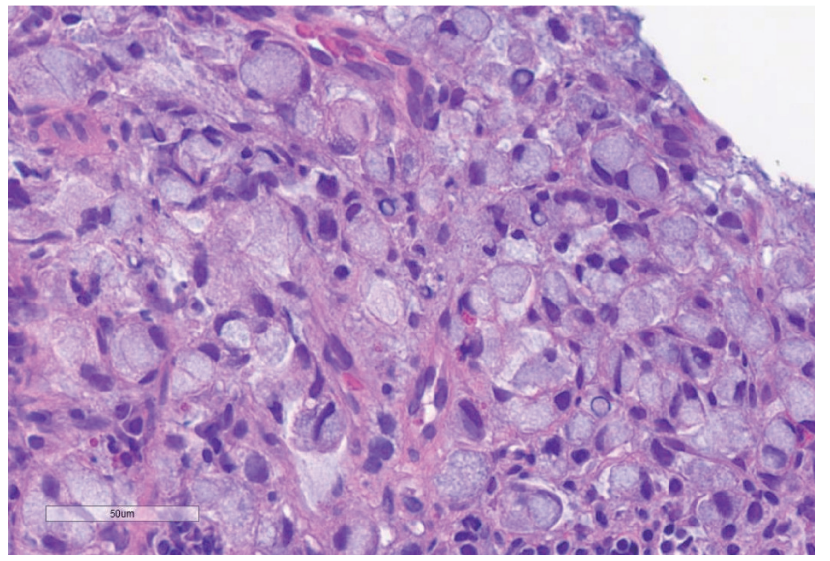

Figure 3. Poorly differentiated adenocarcinoma with signet ring cell features $(\mathrm{H} \& \mathrm{E}$, original $\times 400)$. H\&E: hematoxylin and eosin stain.

staining of the biopsy specimens of the neuroendocrine carcinoma revealed it to be positive for chromogranin synaptophysin, cluster of differentiation (CD)56, cytokeratin (CK)CK 20, while negative for CK7. Immunohistochemistry stain did not show any loss of PTEN protein. Liver biopsy also showed mixed adenocarcinoma and neuroendocrine carcinoma, highgrade (Fig. 7). An attempt at surgical resection of the diseased bowel segment was unsuccessful due to diffuse disease and ileostomy was created. The patient was treated with carboplatin and irinotecan. His liver lesions did not progress and his abdominal disease has not progressed, currently waiting for next gene testing to target the treatment with immunotherapy.

\section{Case 2}

A 43-year-old man presented to the emergency department with lower abdominal pain of 6 weeks duration. He described the pain as intermittent, crampy, involving the lower abdominal quadrants mainly and severe in intensity. It was associated 


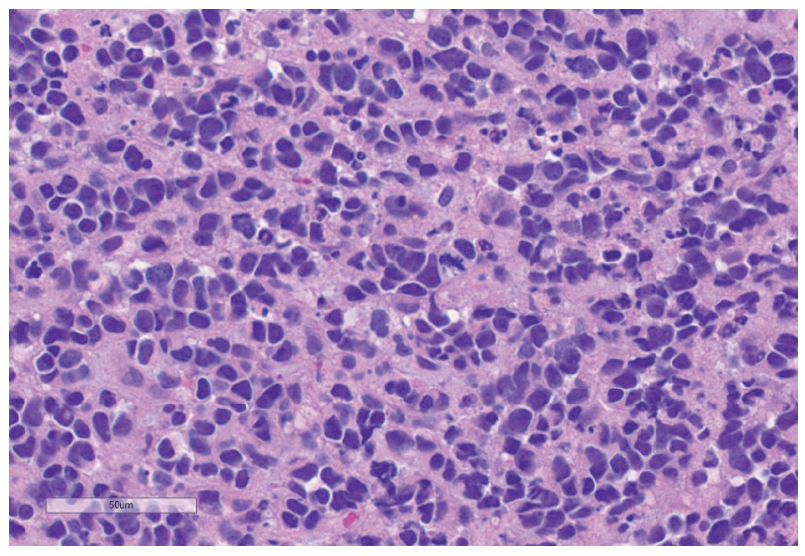

Figure 4. High-grade neuroendocrine carcinoma (H\&E, original $\times 400$ ). H\&E: hematoxylin and eosin stain.

with nausea but no vomiting and a 2-day history of diarrhea. He described the stool to be watery, occurring twice daily, yellowish in color without any blood in it. He had a past medical history significant for arthritis and hypothyroidism. He smoked 1 - 2 cigars per week and denied any history of alcohol or drug use. On initial examination he was afebrile with stable vital signs. His abdominal examination, review of systems and initial laboratory tests were unremarkable. He had a CT abdomen with intravenous contrast revealing focal segmental wall thickening of the transverse colon concerning for neoplasm. He underwent colonoscopy that showed mucosal ulceration in the transverse colon. Histopathology of the biopsy specimens showed focal superficial ulcer, mild acute inflammation, granulation tissue reaction, and reactive glandular atypia. Patient was discharged with outpatient clinic follow-up. On subsequent visits he reported diarrhea that continued for 3 months and did not improve at all. Therefore, he underwent a repeat colonoscopy which showed normal colonic mucosa but noted to have an 8-mm polyp in the ascending colon which was removed with a hot snare. Histopathology of the polyp showed ganglioneuroma. After the second exam, patient reported symptomatic improvement when he was called but did

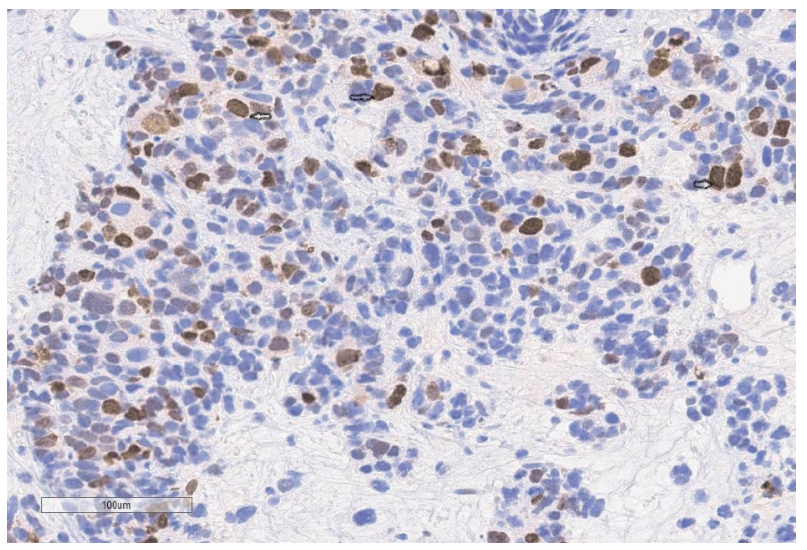

Figure 5. High-grade neuroendocrine carcinoma with Ki67 proliferative index $=40 \%$ (Ki67 immunostain, original $\times 200)$ with multiple black arrows showing KIT positivity.

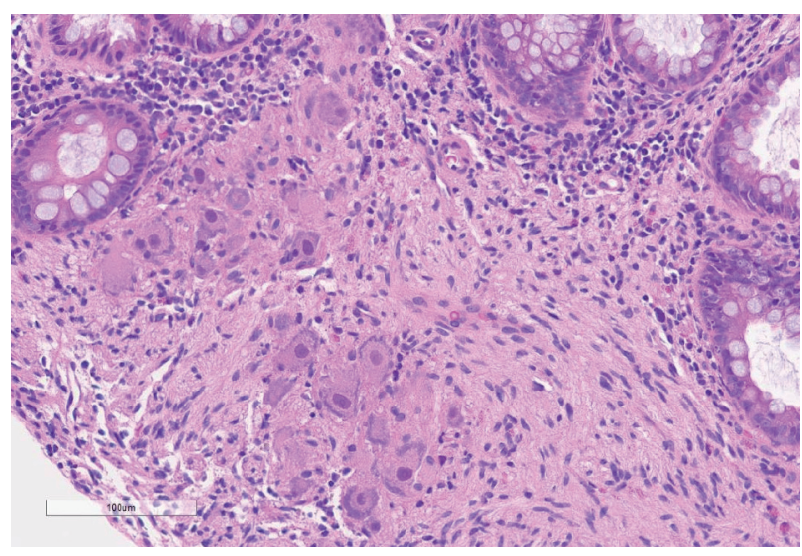

Figure 6. Ganglioneuroma with neuromatous stroma and dispersed ganglion cells $(H \& E$, original $\times 100)$. H\&E: hematoxylin and eosin stain.

not come for further follow-up in clinic.

\section{Discussion}

Ganglioneuromas are neuroectodermal tumors. They are found at various anatomical sites in the body including mediastinum, retroperitoneum, adrenal glands, as well as GI tract, and are rarely found in the colon [6].

Ganglioneuroma has been classified by Shekitka et al into three major groups: polypoid ganglioneuroma; GP; and DG (eight patients) [7].

Polypoid ganglioneuromas are most common and has favorable prognosis. They present as small, sessile or pedunculated polyps, their size ranges from few millimeters to $3 \mathrm{~cm}$. Histologically it resembles the juvenile polyp with disturbed crypt architecture and cystic glands, expanded lamina propria and smooth surface epithelium or nodular mucosal or submucosal ganglion and spindle cells.

GP usually presents as multiple (20 to 40 to innumerable) sessile or pedunculated polyps, their size ranging from $1 \mathrm{~mm}$ to $2.2 \mathrm{~cm}$. Histologically it resembles polypoidal ganglioneu-

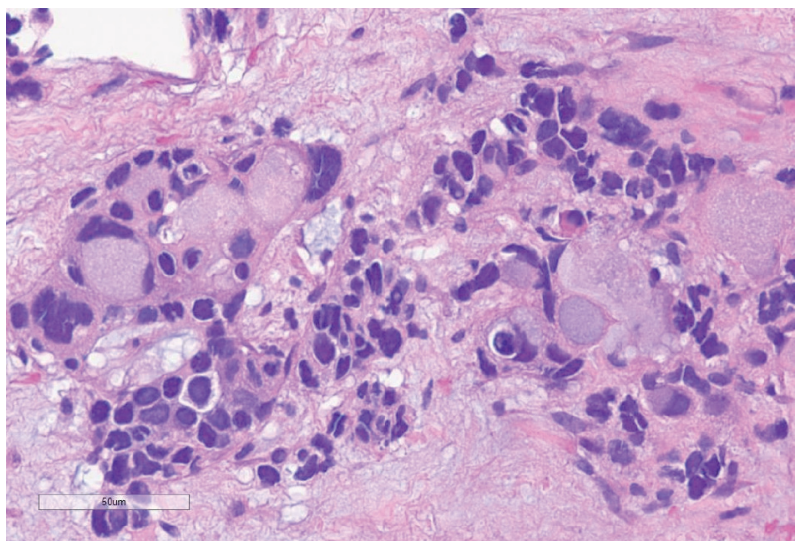

Figure 7. Mixed adenocarcinoma high-grade neuroendocrine carcinoma in metastatic liver lesion (H\&E, original $\times 400)$. H\&E: hematoxylin and eosin stain. 
roma or it may have unusual filiform mucosal projections. It has been described in association with familial adenomatous polyposis (FAP), Cowden's disease, tuberous sclerosis, multiple endocrine neoplasia type $2 \mathrm{~b}$ (MEN type IIb) syndrome, colorectal carcinoma and juvenile polyposis.

DG of the GI tract is a poorly demarcated nodular or transmural proliferation of ganglioneuromatous tissue that diffusely involves the myenteric plexus and has worse prognosis. The lesions are typically large ranging from 1 to $17 \mathrm{~cm}$ in greatest dimension. Histologically it ranges from fusiform, hyperplastic expansion of the myenteric plexus to irregular transmural ganglioneuroma. This is usually associated with neurofibromatosis type 1 (von Recklinghausen disease) or MEN IIb (parathyroid adenoma and medullary carcinoma of the thyroid) [7].

Ganglioneuromas are usually asymptomatic; however depending on the location and size have a wide range of clinical presentations. GI ganglioneuroma may present with abdominal pain, rectal bleeding, irritable bowel syndrome, constipation, diarrhea and megacolon with intestinal obstruction [8-13].

Colonic ganglioneuromas are usually confirmed by colonoscopy with polypectomy. Once the histological diagnosis of ganglioneuroma is confirmed, if it is DG or GP further genetic testing is recommended to screen for associated syndromes (Cowden's disease, von Recklinghausen disease or MEN type IIb) [7, 9, 11].

The management of ganglioneuromas depends on the initial presentation of the patient and associated syndrome. Asymptomatic individuals usually require no treatment and no guidelines endorse surveillance although a few case reports including our first patient showed the coexistence of ganglioneuroma with malignancy. On the other hand, if they are associated with clinical symptoms, management can be modified to target specific symptoms, like intestinal obstruction that may need surgical intervention including resection $[7,10,11,13]$.

\section{Conclusions}

GI ganglioneuroma is extremely rare, with a broad array of clinical presentations depending on the size and location. At times they are associated with genetic syndromes and need further investigations for diagnosis of respective conditions. Although generally viewed as a benign disease, a few cases in literature have pointed out its coexistence with malignancy including similar to our first patient. Currently no clinical guidelines are available for management or surveillance for patients with ganglioneuromas, but it creates a diagnostic and clinical dilemma for physicians, and its varied clinical association should be kept in mind when managing these patients.

\section{Acknowledgments}

None to declare.

\section{Financial Disclosure}

None of the authors have any financial conflict of interest.

\section{Conflict of Interest}

The authors declare that they have no conflict of interest.

\section{Informed Consent}

Patients provided informed consents for publication of this report and the accompanying images.

\section{Author Contributions}

Ahmed Baiomi wrote the initial manuscript; Hafsa Abbas reviewed the images and manuscript; Bhavna Balar, Myrta Daniel critically reviewed and modified the manuscript, Masooma Niazi, Helen Remotti critically reviewed, modified the manuscript and provided the histopathology slides and the legends.

\section{Data Availability}

The authors declare that data supporting the findings of this study are available within the article.

\section{References}

1. Chan OT, Haghighi P. Hamartomatous polyps of the colon: ganglioneuromatous, stromal, and lipomatous. Arch Pathol Lab Med. 2006;130(10):1561-1566.

2. Haggitt RC, Reid BJ. Hereditary gastrointestinal polyposis syndromes. Am J Surg Pathol. 1986;10(12):871-887.

3. Kanter AS, Hyman NH, Li SC. Ganglioneuromatous polyposis: a premalignant condition. Report of a case and review of the literature. Dis Colon Rectum. 2001;44(4):591-593.

4. Macenlle R, Fernandez-Seara J, Pato M, Pereira J, Pascual P, Montero M, Miranda C. Ganglioneuromatous polyposis of the colon associated with adenocarcinoma and primary hyperparathyroidism. Eur J Gastroenterol Hepatol. 1999;11(4):447-450.

5. Snover DC, Weigent CE, Sumner HW. Diffuse mucosal ganglioneuromatosis of the colon associated with adenocarcinoma. Am J Clin Pathol. 1981;75(2):225-229.

6. Fiori E, Pozzessere C, Lamazza A, Leone G, Borrini F, Schillaci A, Mingazzini P. Endoscopic treatment of ganglioneuroma of the colon associated with a lipoma: a case report. J Med Case Rep. 2012;6:304.

7. Shekitka KM, Sobin LH. Ganglioneuromas of the gastrointestinal tract. Relation to Von Recklinghausen disease and other multiple tumor syndromes. Am J Surg Pathol. 1994;18(3):250-257.

8. Soccorso G, Puls F, Richards C, Pringle H, Nour S. A ganglioneuroma of the sigmoid colon presenting as leading point of intussusception in a child: a case report. J Pediatr Surg. 2009;44(1):e17-20. 
9. Smith VV, Eng C, Milla PJ. Intestinal ganglioneuromatosis and multiple endocrine neoplasia type $2 \mathrm{~B}$ : implications for treatment. Gut. 1999;45(1):143-146.

10. Rosenfeld EH, Chumpitazi BP, Castro E, Naik-Mathuria B. Diffuse Intestinal Ganglioneuromatosis Causing Severe Intestinal Dysmotility in a Child With a PTEN Mutation. J Pediatr Gastroenterol Nutr. 2019;68(2):e35-e37.

11. Mitra S, Mukherjee S, Chakraborty H. Isolated ileal ganglioneuromatosis in an 11-year-old boy: Case re- port and review of literature. Indian J Pathol Microbiol. 2016;59(3):365-367.

12. Matthews MA, Adler BH, Arnold MA, Kumar S, Carvalho R, Besner GE. Diffuse intestinal ganglioneuromatosis in a child. J Pediatr Surg. 2013;48(5):1129-1133.

13. Fernando AR, Samarasekera DN, Bulathsinghela RP. Constipation with megacolon in a 36-year-old man: a rare presentation of MEN2B from Sri Lanka. BMJ Case Rep. 2019;12(1):bcr-2018-227081. 